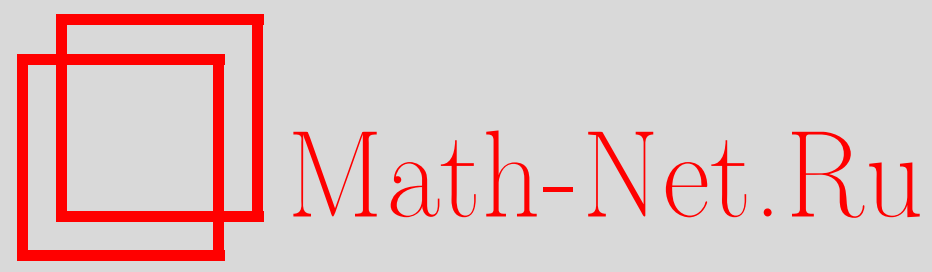

А. П. Терехин, Сферический аналог комплексной формы тригонометрического ряда Фурье; приближение, Матем. заметки, 2000, том 67, выпуск 5, 764-777

DOI: https://doi.org/10.4213/mzm894

Использование Общероссийского математического портала Math-Net.Ru подразумевает, что вы прочитали и согласны с пользовательским соглашением http://www . mathnet.ru/rus/agreement

Параметры загрузки:

IP : 54.209 .52 .79

26 апреля 2023 г., 16:50:03

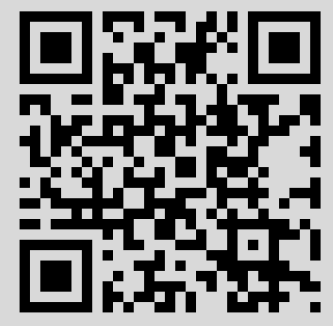




\section{СФЕРИЧЕСКИЙ АНАЛОГ КОМПЛЕКСНОЙ ФОРМЫ ТРИГОНОМЕТРИЧЕСКОГО РЯДА ФУРЬЕ; ПРИБЛИЖЕНИЕ}

\section{А. П. Терехин}

Для функции, определенной на единичной сфере размерности два и вьше, предложен близкий по алгебраической струк туре сферический аналог элементов ряда Фурье функции, определенной на единичной окружности. В различных нормах оценено уклонение от функции аналогов частичных сумм Фурье.

Библиография: 2 названия.

В статье решаются такие две задачи.

1. Для функций, определенных на единичной сфере размерности два и выше, построить близкий по алгебраической структуре сферический аналог элемента тригонометрического ряда Фурье функций, определенных на единичной окружности; от аналога требуется лишь, чтобы он был, по крайней мере, сферическим полиномом (алгебраическим многочленом на сфере), но не обязательно сферической гармоникой.

2. Выяснить условия сходимости и оценить в различных нормах уклонение от функции сумм ее сферических элементов Фурье.

\section{1. Сферический аналог элементов ряда Фурье}

Целевые формулы. Несомненно влияние поля комплексных чисел на теорию тригонометрических рядов Фурье. То, что оно дает для теории и что мы намерены перенести на случай сферы, можно увидеть из следующего представления тригонометрической составляющей функции $f$, определенной на единичной окружности $C$ :

$$
\frac{1}{2 \pi} \int_{C} f(\zeta)\left(z \zeta^{-1}\right)^{m} d \zeta, \quad m \in \mathbb{Z} .
$$

Как мы видим, здесь используются существование обратного для каждого вектора единичной окружности, умножение ее векторов и умножение вектора на скаляр или, если функция векторнозначная, на вектор того линейного пространства над полем комплексных чисел, где функция принимает свои значения. Лишш структуры линейного пространства на плоскости достаточно для представления

$$
\zeta=z \cos \theta+z^{\perp} \sin \theta
$$

произвольного вектора $\zeta \in C$ через вектор $z \in C$ и ему ортогональный $z^{\perp}$; для всякого $\zeta \in C$ единственным образом на отрезке $[0, \pi]$ определяется $\theta=\arccos (\zeta, z)$, где $(\zeta, z)-$ 
скалярное произведение; тогда для $\zeta \neq \pm z$ единственным образом определится $z^{\perp}$ как тот вектор из двух, для которого $\left(\zeta, z^{\perp}\right)>0$.

Существование обратного $z^{-1}$ позволяет от представления (2) перейти к представлению

$$
\zeta z^{-1}=\cos \theta+z^{\perp} z^{-1} \sin \theta
$$

с использованием лиш структуры алгебры (гиперкомплексной системы) поля комплексных чисел, без использования коммутативности и даже ассоциативности умножения.

Решающими для “комплексности” представления (3) являются равенства

$$
\left|z^{\perp} z^{-1}\right|=1, \quad\left(z^{\perp} z^{-1}\right)^{2}=-1
$$

в случае комплексных чисел они тривиальны, так как $z^{\perp} z^{-1}=z \exp ( \pm i \pi / 2) z^{-1}= \pm i$.

Коммутативность и ассоциативность умножения комплексных чисел позволяют отделить в представлении (1) коэффициент Фурье функции от степени $z^{m}$. Последняя является однородньм многочленом от координат вектора $z$. Сугубо аналитичность делает степень гармонической.

В пространствах большей размерности (трех и вьше), как известно, уже нет возможностей поля комплексных чисел. Здесь ставим задачу воспользоваться такой алгебраической структурой в пространстве, которая позволила бы иметь аналоги формул (3) и (4). Требуем еще, чтобы аналог элемента (1) давал, по крайней мере, алгебраический многочлен от координат вектора, который будет стоять на месте $z$.

Обозначения. $d \in\{3,4, \ldots\}, \sigma$ - единичная сфера пространства $\mathbb{R}^{d},(\nu, \mu)$ - скалярное произведение, $\sigma(\mu):=\{\nu \in \sigma \mid(\nu, \mu)=0\}$ - экватор сферы с полюсом $\mu,|\sigma|-$ мера (объем) сферы.

Переменная единица. В общем случае пространства возможен такой подход к решению поставленной задачи, в алгебраической части в общем-то известньй.

Взяв на роль $z$ из (1) вектор $\mu \in \sigma$ и взяв затем вектор $\nu \in \sigma(\mu)$, наделим плоскость $\Pi_{\nu}$ векторов $\mu$ и $\nu$ структурой поля комплексных чисел, объявив $\mu$ единицей (общей для всех плоскостей $\Pi_{\nu}$ ), а $\nu$ - мнимой единицей поля (таким образом, $\Pi_{\nu}$ и $\Pi_{-\nu}-$ два комплексных поля одной плоскости).

Поскольку каждый вектор $x \in \mathbb{R}^{d}$ принадлежит или “действительной” прямой вектора $\mu$ или, в ином случае, единственной своей “комплексной” плоскости $\Pi_{\nu} \mathrm{c}(x, \nu)>0$, то для $x \neq 0$ будут существовать обратный вектор $x^{-1}$ и его степени $\left(x^{-1}\right)^{m}, m \in \mathbb{Z}$; последние можно обозначить как $\left(\mu x^{-1}\right)^{m}$, отмечая в обозначении зависимость от единищы $\mu$; зависимость от мнимой единицы $\nu$ показьвает вектор $x$, ее определяющий.

Будем иметь следующие аналоги формул (1)-(4):

$$
\begin{gathered}
\frac{1}{|\sigma|} \int_{\sigma} f(\xi)\left(\mu \xi^{-1}\right)^{m} d \xi, \\
\xi=\mu \cos \theta+\nu \sin \theta, \quad \nu \in \sigma(\mu), \\
\nu^{2}=-\mu .
\end{gathered}
$$


Интеграл $\left(1^{\prime}\right)$ является алгебраическим многочленом от координат вектора $\mu$. Действительно, в $\left(2^{\prime}\right)$ имеем $\cos \theta=(\mu, \xi), \nu \sin \theta=\xi-\mu(\mu, \xi)$, и следовательно,

$\xi^{m}=\mu \cos m \theta+\nu \sin m \theta=\mu \cos m \theta+(\xi-\mu(\mu, \xi)) \frac{\sin m \theta}{\sin \theta}=\frac{-\mu \sin (m-1) \theta+\xi \sin m \theta}{\sin \theta}$.

Осталось учесть, что отношения $\sin n \theta / \sin \theta$ суть многочлены от $\cos \theta=(\mu, \xi)$. Они не являются в общем случае однородными; так, например, $\sin 3 \theta / \sin \theta=4(\mu, \xi)^{2}-1$.

Можно проверить, что $\Delta \xi^{m}=-\mu v_{m-1}(\theta)+\xi\left(v_{m}(\theta)+2 u_{m-1}(\theta)\right)$, где $u_{n}(\theta)=$ $(\sin n \theta / \sin \theta)^{\prime} / \sin \theta, v_{n}(\theta)=u_{n}^{\prime}(\theta) / \sin \theta$. Таким образом, гармоничности степеней $\xi^{m}$ также нет.

Общая единица. Более близкий аналог по сравнению с тем, что дает переменная единица, давала бы алгебра с единицей, общей для пространства ( и сохранившая аналоги целевых формул (3), (4)).

Аналог представления (3) следует из общего для всех пространств представления $\left(2^{\prime}\right)$ при наличии в алгебре обратного элемента, что предполагают аналоги целевых формул (3) и (4):

$$
\xi \mu^{-1}=\cos \theta+\nu \mu^{-1} \sin \theta
$$

$\xi, \mu \in \sigma, \nu \in \sigma(\mu)$ (обозначения в начале предыдущего пункта).

Аналоги равенств (4) будут выглядеть следующим образом:

$$
\left|\nu \mu^{-1}\right|=1, \quad\left(\nu \mu^{-1}\right)^{2}=-1 \quad(\mu \in \sigma, \quad \nu \in \sigma(\mu)) .
$$

При $\mu=1$ второе из равенств $\left(4^{\prime \prime}\right)$ дает $\iota^{2}=-1$ для всех $\iota \in \sigma(1)$.

Положив, пользуясь представлением $\left(2^{\prime}\right), \nu \mu^{-1}=\cos \varphi+\iota \sin \varphi, \iota \in \sigma(1)$ (учли, что $\nu \mu^{-1} \in \sigma$ в силу первого из равенств $\left.\left(4^{\prime \prime}\right)\right)$, будем иметь $-1=\left(\nu \mu^{-1}\right)^{2}=\cos 2 \varphi+\iota \sin 2 \varphi$, откуда $2 \varphi=\pi$ и, следовательно, $\nu \mu^{-1}=\iota \in \sigma(1)$. Но тогда ввиду $\left(3^{\prime \prime}\right)\left|\xi \mu^{-1}\right|=1$ для всех $\xi, \mu \in \sigma$. Последнее означает, что алгебра необходимо должна быть нормированной $(|x y|=|x||y|)$, что по известной теореме Гурвица возможно лишь в пространствах размерностей $1,2,4,8$.

В интересующих нас случаях (4 и 8) такие алгебры, как известно, образуют соответственно тела кватернионов и октав Кэли. В этих алгебрах очевидным образом имеют место представление $\left(3^{\prime \prime}\right)$ и первое из равенств $\left(4^{\prime \prime}\right)$ (ввиду нормированности); второе равенство в $\left(4^{\prime \prime}\right)$ следует из того факта, что минус единицу дает квадрат единичного (по норме) "мнимого" вектора, каковым является произведение $\nu \mu^{-1} \mathrm{c} \nu \in \sigma(\mu)$ : $\nu \mu^{-1}=\left(y_{0}+y\right)\left(x_{0}-x\right)=-y_{0} x+x_{0} y+y_{0} x_{0}-y x, y x=-(y, x)+[y, x], x_{0}$ - “действительные" части векторов $\mu$ (и $\nu$ соответственно), $x$ - их “мнимые" части, скобки в последнем равенстве означают соответственно скалярное и векторное произведения; “мнимость" $\nu \mu^{-1}$ следует из равенства $y_{0} x_{0}+(y, x)=(\nu, \mu)=0$.

Что касается других пространств $\mathbb{R}^{d}$, то при $d=3,5,6,7$ их можно погрузить в $\mathbb{R}^{4}$ или $\mathbb{R}^{8}$, считая векторы из $\mathbb{R}^{d}$ "мнимьми" (произведению векторов из $\mathbb{R}^{d}$ придется разрешить выход из $\left.\mathbb{R}^{d}\right)$. Ясно, что равенства $\left(3^{\prime \prime}\right)$ и $\left(4^{\prime \prime}\right)$ сохраняются (теперь уже для $\left.\mu \in \sigma \subset \mathbb{R}^{d}, \nu \in \sigma(\mu) \subset \mathbb{R}^{d}\right)$.

Наконец, для названных пространств будет вьполнено и последнее требование - чтобы аналог (1') давал алгебраический (векторный) многочлен от координат вектора $\mu$. 
Действительно, в любой алгебре (гиперкомплексной системе) произведение является линейной функцией координат каждого сомножителя, поэтому и в алгебре кватернионов и октав степень $\left(\mu \xi^{-1}\right)^{m}$ будет алгебраическим многочленом порядка $|m|$ от координат вектора $\mu$, причем многочленом однородным. Заметим еше, что поскольку для кватернионов и октав $\xi \mu^{-1}=\left(\mu \xi^{-1}\right)^{-1}$, то в аналоге $\left(1^{\prime}\right)$ равноправны $\mu \xi^{-1}$ и $\xi \mu^{-1}$.

Как и в случае с переменной единицей, можно проверить, что

$$
\Delta\left(\xi \mu^{-1}\right)^{m}=-m u_{m}(\theta)+\nu \mu^{-1} u_{m}^{\prime}(\theta),
$$

т. е. степень не гармонична.

\section{2. Сферические аналоги сумм Фурье}

Обозначения. $r:=d-2$, где $d$ - размерность пространства; $\left|\sigma^{\prime}\right|$ - мера (объем) экватора сферы $\sigma$ (см. также обозначения п. 2).

Равенства $\left(3^{\prime}\right),\left(4^{\prime}\right)$ и $\left(3^{\prime \prime}\right),\left(4^{\prime \prime}\right)$ позволяют воспользоваться формулой Моавра, в силу которой соответственно

$$
\xi^{m}=\mu \cos m \theta+\nu \sin m \theta, \quad\left(\xi \mu^{-1}\right)^{m}=\cos m \theta+\nu \mu^{-1} \sin m \theta .
$$

Тогда

$$
\sum_{m=-n}^{n} \xi^{m}=2 \mu D_{n}(\breve{\mu \xi}), \quad \sum_{m=-n}^{n}\left(\xi \mu^{-1}\right)^{m}=2 D_{n}(\breve{\mu \xi})
$$

где $\widetilde{\mu \xi}=\theta$,

$$
D_{n}(\theta)=\frac{1}{2}+\sum_{m=1}^{n} \cos m \theta=\frac{\sin (n+1 / 2) \theta}{2 \sin (\theta / 2)}
$$

- ядро Дирихле.

Таким образом, соответствующая сумма сферических элементов Фурье (см. (1')) будет иметь вид

$$
\frac{2}{|\sigma|} \int_{\sigma} f(\xi) D_{n}(\stackrel{\mu}{\mu}) d \xi
$$

(в случае переменной единищы последняя как множитель опущена). Заметим, что сумма элементов не зависит от выбора единицы.

В связи с вопросом о сходимости сумм к функции подсчитаем представляющий сумму интеграл для $f=1$. Используем с этой целью формулу сведения сферического интеграла к повторным

$$
\int_{\sigma} f(\xi) d \xi=\int_{0}^{\pi} \int_{\sigma(\mu)} f(\mu \cos \theta+\nu \sin \theta) d \nu \sin ^{r} \theta d \theta
$$

Получаем

$$
\int_{\sigma} D_{n}(\widetilde{\mu \xi}) d \xi=\left|\sigma^{\prime}\right| \int_{0}^{\pi} D_{n}(\theta) \sin ^{r} \theta d \theta
$$

При четном $r$ и $n \geqslant r$ интеграл (8) равен нулю, поскольку $\sin ^{r} \theta$ является четным тригонометрическим полиномом порядка не выше $n$, а (двойной) интеграл равен значению такого полинома в нуле. 
2.1. При нечетном $r$ будем иметь

$$
D_{n}(\theta) \sin ^{r} \theta=\sin \left(n+\frac{1}{2}\right) \theta \cos \frac{\theta}{2} \sin ^{r-1} \theta=\frac{1}{2}(\sin (n+1) \theta+\sin n \theta) \sin ^{r-1} \theta .
$$

Обозначая через $2 m+1$ нечетное из чисел $n+1$ или $n$, находим

$$
\int_{0}^{\pi} D_{n}(\theta) \sin ^{r} \theta d \theta=\frac{1}{2} \int_{0}^{\pi} \sin ^{r-1} \theta \sin (2 m+1) \theta d \theta
$$

так как интеграл с другим синусом равен нулю (см., например, [1, с. 386]). Там жеможно найти значение последнего интеграла - с учетом $1 / 2$ он равен

$$
\frac{1}{\varkappa_{n}}=(-2)^{(r-1) / 2} \frac{((r-1) / 2) !(r-2) ! !(2 m-r) ! !}{(2 m+r) ! !}
$$

(при этом $(-1) ! !=1)$. Так как $(2 m+r) ! ! /(2 m-r) ! !=\prod_{k=1}^{r}(2 m-r+2 k)$, ясно, что интеграл (8) при нечетном $r$ имеет порядок $n^{-r}$.

Таким образом, полагая

$$
S(t) f(\mu):=\frac{1}{\left|\sigma^{\prime}\right|} \int_{\sigma(\mu)} f(\mu \cos t+\nu \sin t) d \nu
$$

получим следующее представление нормированной суммы элементов Фурье функции $f$ :

$$
D_{n}(f, \mu):=\frac{\varkappa_{n}}{\left|\sigma^{\prime}\right|} \int_{\sigma} f(\xi) D_{n}(\widetilde{\mu}) d \xi=\varkappa_{n} \int_{0}^{\pi} S(\theta) f(\mu) D_{n}(\theta) \sin ^{r} \theta d \theta
$$

где $\varkappa_{n}$ определено равенством (9) из условия $D_{n}(1, \mu)=1$ и имеет порядок $n^{r}$.

2.2. При четном $r$ ввиду нулевого значения интеграла (8) нормирование полинома (6) только числовым множителем невозможно.

2.2.1. Нормируем интеграл (6) таким образом, чтобы

$$
\frac{\varkappa}{\left|\sigma^{\prime}\right|} \int_{\sigma} D_{n}(\widetilde{\mu \xi})\left(\frac{\sin m \mu \xi}{\sin \widetilde{\mu \xi}}\right)^{r} d \xi=1
$$

и полагаем

$$
\begin{aligned}
D_{n, m}(f, \mu) & :=\frac{\varkappa}{\left|\sigma^{\prime}\right|} \int_{\sigma} f(\xi) D_{n}(\widetilde{\mu \xi})\left(\frac{\sin m \mu \xi}{\sin \mu \xi}\right)^{r} d \xi \\
& =\varkappa \int_{0}^{\pi} S(\theta) f(\mu) D_{n}(\theta) \sin ^{r} m \theta d \theta
\end{aligned}
$$

(снова воспользовались формулой (7)). В силу четности $r$ это будет сферический полином; порядок его не выше $n+r(m-1)$. 
Задача теперь состоит в подборе по $n$ u $r$ натурального параметра $m$ такого, чтобы

$$
\int_{0}^{\pi} D_{n}(\theta) \sin ^{r} m \theta d \theta \neq 0
$$

Ясно, что должно быть $r m>n$ (аргументацию см. вьше после (8)).

Учитывая, что

$$
\sin ^{r} m \theta=2^{-r}\left(C_{r}^{r / 2}+2 \sum_{k=1}^{r / 2}(-1)^{k} C_{r}^{r / 2-k} \cos 2 k m \theta\right)
$$

имеем

$$
\begin{aligned}
\frac{2}{\pi} \int_{0}^{\pi} D_{n}(\theta) \sin ^{r} m \theta d \theta & =2^{-r}\left(C_{r}^{r / 2}+2 \sum_{2 k m \leqslant n}(-1)^{k} C_{r}^{r / 2-k}\right) \\
& =-2^{-r+1} \sum_{2 k m>n}(-1)^{k} C_{r}^{r / 2-k}
\end{aligned}
$$

- в заключение учли, что полная сумма коэффициентов равна нулю (аргументация там же - после (8)).

Так как необходимо $r m>n$, то последняя сумма не пуста, а поскольку биномиальные коэффициенты $C_{2 p}^{p-k}$ с ростом $k$ до $p$ строго убывают, эта сумма и не нулевая. Возможность нормирования доказана. Кроме того, как это вытекает из (13), чтобы в $\left(11^{\prime}\right)$ иметь $D_{n, m}(1, \mu)=1$, следует положить

$$
\varkappa=-(-1)^{r / 2} \frac{2^{r}}{\pi}
$$

при условии $(r-2) m \leqslant n$, когда в сумму (13) не будет входить слагаемое с $k=r / 2-1$; таким образом, общим условием равенства (14) будет

$$
(r-2) m \leqslant n<r m
$$

Задача выбора по $n$ и $r$ подходящих $m$ и $\varkappa$ решена.

Следуюшим шагом проанализируем представление $\left(11^{\prime}\right)$.

С учетом (12) и (5) будем иметь

$$
D_{n}(\theta) \sin ^{r} m \theta=2^{-r}\left(C_{r}^{r / 2} D_{n}(\theta)+\sum_{k=1}^{r / 2}(-1)^{k} C_{r}^{r / 2-k} \sin \left(n+\frac{1}{2}\right) \theta \frac{\cos 2 k m \theta}{\sin (\theta / 2)}\right) .
$$

Но $2 \sin (n+1 / 2) \theta \cos 2 k m \theta=\sin (n+2 k m+1 / 2) \theta+\sin (n-2 k m+1 / 2) \theta$; следовательно,

$$
\begin{aligned}
2^{r} D_{n} \sin ^{r} m \theta= & C_{r}^{r / 2} D_{n}+\sum_{k=1}^{r / 2-1}(-1)^{k} C_{r}^{r / 2-k}\left(D_{n+2 k m}+D_{n-2 k m}\right) \\
& +(-1)^{r / 2}\left(D_{n+r m}-D_{r m-n}\right) .
\end{aligned}
$$


В обозначении номеров ядер Дирихле учли условие (15), в силу которого $r m-n>0$ и $n-2 k m \geqslant 0$ для всех $k$ от 1 до $r / 2-1$.

Таким образом, представление $\left(11^{\prime}\right)$ преобразуется в следующее:

$$
\begin{aligned}
D_{n, m}(f, \mu)= & -\frac{(-1)^{r / 2}}{\pi} \int_{0}^{\pi} S(\theta) f(\mu)\left(C_{r}^{r / 2} D_{n}(\theta)\right. \\
& +\sum_{k=1}^{r / 2-1}(-1)^{k} C_{r}^{r / 2-k}\left(D_{n+2 k m}(\theta)+D_{n-2 k m}(\theta)\right) \\
& \left.+(-1)^{r / 2}\left(D_{n+r m}(\theta)-D_{r m-n}(\theta)\right)\right) d \theta
\end{aligned}
$$

при этом $D_{n, m}(1, \mu)=1$.

Обозначим через $S_{n}(\varphi, t)$ частичные суммы классического тригонометрического ряда $\Phi$ урье функции $\varphi$ действительного переменного $t \in \mathbb{R}$, через $S f(\mu)-$ функцию того же действительного переменного, определяемую равенством $S f(\mu)(t)=S(t) f(\mu)$. В этих обозначениях представление (16) можно записать компактнее:

$$
D_{n, m}(f, \mu)=\sum c_{k} S_{k_{n}}(S f(\mu), 0)
$$

где коэффищиенты $c_{k}$ и номера $k_{n}$ определяются из предыдущего представления.

2.2.2. Выбор $m$ и $n$ по заданному порядку полинома. Пусть $n \in \mathbb{Z}_{0}$. Выберем по $n$ целые $n_{r}$ и $m_{r}$ на место прежних $n$ и $m$ в $\left(11^{\prime}\right)$ из следующих условий:

$$
n=n_{r}+r\left(m_{r}-1\right)
$$

т. е. чтобы порядок сферического полинома был не более $n$;

$$
n_{r}-(r-2) m_{r} \simeq r m_{r}-n_{r} \rightarrow \infty
$$

т. е. чтобы наименъшие порядки ядер Дирихле в (16) были по возможности равными и бесконечно большими при $n \rightarrow \infty$. Тогда будет обеспечено названноевыше условие (15) выбора $m_{r}$ : чтобы $r m_{r}>n_{r}>(r-2) m_{r}$.

Попытаемся найти не только достаточные, но и необходимые условия для оптимального нестрогого равенства в (18).

Введем, пока неопределенные, параметры $n_{1}$ и $n_{2}$ и положим

$$
n_{r}-(r-2) m_{r}=n_{1}, \quad r m_{r}-n_{r}=n_{2},
$$

откуда

$$
2 m_{r}=n_{1}+n_{2}, \quad n_{r}=\frac{r\left(n_{1}+n_{2}\right)}{2}-n_{2} .
$$

Ясно, что $n_{1}$ и $n_{2}$ должны иметь общую четность; положим

$$
n_{1}=2 p_{1}+\omega, \quad n_{2}=2 p_{2}-\omega,
$$


где $\omega=0$ или $\omega=1$.

Имеем

$$
m_{r}=p_{1}+p_{2}, \quad n_{r}=r\left(p_{1}+p_{2}\right)-2 p_{2}+\omega .
$$

Удовлетворим требованию (17):

$$
n=r\left(p_{1}+p_{2}\right)-2 p_{2}+\omega+r\left(p_{1}+p_{2}-1\right)=r 2 p_{1}+2(r-1) p_{2}+\omega-r .
$$

Поскольку $r$ четно, $\omega$ должно иметь четность $n$; пусть $n=2 p+\omega$. Тогда

$$
p=r p_{1}+(r-1) p_{2}-\frac{r}{2}
$$

(пришли к линейному диофантову уравнению). Найдем $p_{1}$ и $p_{2}$.

Последнее уравнение равносильно такому:

$$
p+\frac{r}{2}=(r-1)\left(p_{1}+p_{2}\right)+p_{1} .
$$

Пусть

$$
p+\frac{r}{2}=(r-1) q+s, \quad 0 \leqslant s<r-1
$$

(т.е. $s$ - остаток при делении $p+r / 2$ на $r-1$ ).

Разбив $q$ на неопределенные слагаемые, $q=q_{1}+q_{2}$, положим

$$
p_{1}=(r-1) q_{1}+s, \quad p_{2}=q_{2}-(r-1) q_{1}-s
$$

- второе равенство является следствием первого: $p_{2}=\left(p_{1}+p_{2}\right)-p_{1}=q_{2}-p_{1}$.

Удовлетворим требованию приближенного равенства (18), учитьвая (19) и (20):

$$
2 p_{1}+\omega+2 t=2 p_{2}-\omega
$$

где $2 t$ - погрешность равенства (18); ясно, что она должна быть четной. Последнее равенство равносильно такому: $p_{1}+\omega+t=p_{2}$. Подставив в него значения $(23)$, получим

$$
(r-1) q_{1}+s+\omega+t=q_{2}-(r-1) q_{1}-s,
$$

откуда $q_{2}=(2 r-1) q_{1}+2 s+\omega+t$.

Учитывая последнее и выбор $q_{1}$ и $q_{2}$, имеем

$$
q=q_{1}+q_{2}=q_{1}+2(r-1) q_{1}+2 s+\omega+t=(2 r-1) q_{1}+2 s+\omega+t .
$$

Здесь согласно разложению $(22) 2 s+\omega<2(r-1)+1=2 r-1$. Таким образом, $q_{1}-$ результат деления $q$ на $2 r-1$ с остатком $s_{1}=2 s+\omega+t$, откуда определяем

$$
t=s_{1}-2 s-\omega
$$


Итак, по $n$ определяем $p=[n / 2]$ и $\omega=n-2 p$. Находим $q=[(p+r / 2) /(r-1)]$ и $s=p+r / 2-(r-1) q$. Наконец, получаем $q_{1}=[q /(2 r-1)]$ и $s_{1}=q-(2 r-1) q_{1}$.

Полагаем $q_{2}=q-q_{1}$; тогда согласно $(22)$

$$
p_{1}=(r-1) q_{1}+s, \quad p_{2}=q-q_{1}-(r-1) q_{1}-s=q-r q_{1}-s
$$

наконец, по формулам (21) находим

$$
\begin{aligned}
m_{r} & =(r-1) q_{1}+s+q-r q_{1}-s=q-q_{1}, \\
n_{r} & =r\left(q-q_{1}\right)-2\left(q-r q_{1}-s\right)+\omega=(r-2) q+r q_{1}+2 s+\omega .
\end{aligned}
$$

Погрешность в (18), определяемая равенством

$$
2 t:=\left(n_{r}-(r-2) m_{r}\right)-\left(r m_{r}-n_{r}\right)
$$

ввиду (24) равна $2\left(s_{1}-2 s-\omega\right)$.

Далее, $n_{r}-(r-2) m_{r}=(r-2) q+r q_{1}+2 s+\omega-(r-2)\left(q-q_{1}\right)=2(r-1) q_{1}+2 s+\omega \rightarrow \infty$. Требование (18) удовлетворено полностью.

Наконец, $n_{r}+r\left(m_{r}-1\right)=(r-2) q+r q_{1}+2 s+\omega+r\left(q-q_{1}-1\right)=2(r-1) q+2 s+\omega-r=$ $2 p+r+\omega-r=n-$ учли (22) и представление $n=2 p+\omega$. Удовлетворили и требованию (17).

\section{3. Приближение}

3.1. Четномерный случай. Пусть $r$ - четно. Будем пользоваться представлениями (16) и $\left(16^{\prime}\right)$ с переобозначенными $n$ и $m$ на $n_{r}$ и $m_{r}$, определенными по $n$ согласно п. 2.2.2. Сферические полиномы $D_{n, m}(f, \mu)$ с замененными $n$ и $m$ обозначаем через $D_{n}(f, \mu)$. Это соглашение без дополнительных напоминаний будет действовать на протяжении всего настоящего пункта 3.1.

ТЕорема 1 (о сходимости в точке). Ecлu $S_{n}(S f(\mu), 0) \rightarrow f(\mu), \operatorname{mo} D_{n}(f, \mu) \rightarrow f(\mu)$ (обозначения см. выше после $\left(16^{\prime}\right)$ ).

ДокАЗАТЕЛЬСТво. Из представления $\left(16^{\prime}\right)$ следует

$$
D_{n}(d, \mu)-f(\mu)=\sum c_{k}\left(S_{k_{n}}(S f(\mu), 0)-f(\mu)\right)
$$

где $k_{n} \rightarrow \infty$. Далее очевидно.

Пусть теперь $\|f\|$ - норма функций, определенных на сфере; основное требование к норме - сильная непрерывность относительно нее операторной функиии $S(t)$ (см. (10)); такому требованию удовлетворяют нормы пространств $L_{p}(\sigma), 1 \leqslant p<\infty$, и $C(\sigma)$ соответственно функций, интегрируемых с $p$-й степенью и непрерывных на $\sigma$ функций.

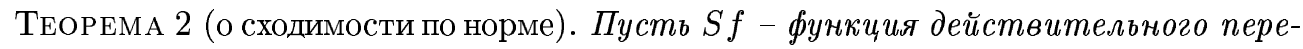
менного, определяемая равенством $(S f)(t)=S(t) f$.

Eсли $S_{n}(S f, 0) \rightarrow f$, то $D_{n}(f) \rightarrow f$ (сходимость по норме). 
ДокАЗАТЕЛЬСТво получаем из того же представления (17), если в нем перейти к норме.

ТЕорема 3 (аналог неравенства Лебега). Имеем

$$
\left\|f-D_{n}(f)\right\| \leqslant C_{r} E_{\underline{n}}(f) \ln (n+2),
$$

әде $E_{n}(f)$ - наилучшее приближение функиии $f$ сферическими полиномами порядка не выше $n, \underline{n}=\min \left\{n_{r}-(r-2) m_{r}, r m_{r}-n_{r}\right\}$; константа $C_{r}$ зависит только от $r$.

ДокАЗАТЕльство. Пусть $T_{\underline{n}}-$ сферический полином наилучшего для $f$ приближения: $\left\|f-T_{\underline{n}}\right\|=E_{\underline{n}}(f)$. Имеем $\bar{D}_{n}\left(T_{\underline{n}}\right)=T_{\underline{n}}$, что следует из представления (17) ввиду того, что $k_{n} \geqslant \underline{n}, S(t) T_{\underline{n}}-$ тригонометрический полином того же порядка, что и сферический полином $T_{\underline{n}}$, и поэтому $S_{k_{n}}\left(S T_{\underline{n}}, 0\right)=S(0) T_{\underline{n}}=T_{\underline{n}}$. Тогда аналогично классическому тригонометрическому случаю будем иметь $D_{n}(f)-f=T_{\underline{n}}-f+D_{n}\left(f-T_{\underline{n}}\right)$. Переходя здесь к нормам, имея в виду $\left(16^{\prime}\right)$, получаем

$$
\left\|D_{n}(f)-f\right\| \leqslant\left\|T_{\underline{n}}-f\right\|\left(1+\sum\left|c_{k}\right|\left\|S_{k_{n}}\left(T_{\underline{n}}-f\right)\right\|\right) .
$$

Но $\left\|S_{k_{n}}\right\| \leqslant L_{k_{n}}$, где $L_{n}-$ константа Лебега (норма в $L$ ядра Дирихле). Осталось учесть, что $L_{n}$ имеет порядок $\ln (n+2)$.

ЗАмечАния. 1. Поповоду констант Лебега см., например, [2, с. 482]; там же см. оценки наилучших приближений (с. 275).

2. В связи с функциональными значениями функции $S f$ и саму функцию $f$ можно считать векторнозначной со значениями в некотором банаховом пространстве с нормой $|\cdot|$. Замечание относится и к нижеследующему случаю нечетномерного пространства.

3.2. Нечетномерный случай. Пусть $r$-нечетно.

ТЕорема 4 (о сходимости в точке). Если в точке $\mu \in \sigma$ средняя $S(\theta) f(\mu)$ как функиия от $\theta \in[0, \pi]$ имеет абсолютно непрерывную производную порядка $r-1$, mo $D_{n}(f, \mu) \rightarrow f(\mu)$.

ДокАЗАТЕЛЬСТВо. В силу (11) и выбора $\varkappa_{n}$

$$
D_{n}(f, \mu)-f(\mu)=\varkappa_{n} \int_{0}^{\pi}(S(\theta) f(\mu)-f(\mu)) D_{n}(\theta) \sin ^{r} \theta d \theta .
$$

Но $D_{n}(\theta) \sin ^{r} \theta=\sin (n+1 / 2) \theta \cos (\theta / 2) \sin ^{r-1} \theta$ (представление ядра Дирихле $D_{n}$ см. в (17)). Полагая

имеем

$$
\Phi(\theta)=(S(\theta) f(\mu)-f(\mu)) \cos \left(\frac{\theta}{2}\right) \sin ^{r-1} \theta,
$$

$$
D_{n}(f, \mu)-f(\mu)=\varkappa_{n} \int_{0}^{\pi} \Phi(\theta) \sin \left(n+\frac{1}{2}\right) \theta d \theta .
$$

Интегрируя $r$ раз по частям, получаем

$$
D_{n}(f, \mu)-f(\mu)=\frac{\varkappa_{n}}{(n+1 / 2)^{r}} \int_{0}^{\pi} \Phi^{(r)}(\theta) \sin \left(\left(n+\frac{1}{2}\right) \theta-\frac{r \pi}{2}\right) d \theta
$$

(подстановки пределов интегрирования дадут нулевые значения: первые за счет $\sin \theta$, последние - за счет $\cos (\theta / 2)$ в $\pi$ и разности $S(\theta) f(\mu)-f(\mu)$ в 0$)$. Так как $\varkappa_{n} \asymp n^{r}$ и производная суммируема, последний интеграл, а с ним и разность $D_{n}(f, \mu)-f(\mu)$ стремятся к нулю. 
Лемма. Пусть $s \in \mathbb{Z}_{0} ; k, m \in \mathbb{N} ; \widetilde{\Delta}(h) \varphi(\theta)=\varphi(\theta+h)-\varphi(\theta-h), \widetilde{\Delta}^{k}(h)-$ степень

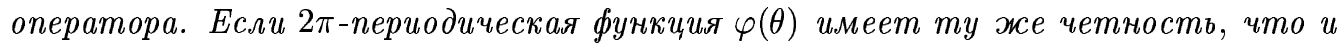
$\cos (\theta-s \pi / 2)$, mo

$$
\int_{0}^{\pi} \varphi(\theta) \cos \left(m \theta-\frac{s \pi}{2}\right) d \theta=2^{-k} \int_{0}^{\pi}\left(\widetilde{\Delta}^{k}\left(\frac{\pi}{2 m}\right) \varphi(\theta)\right) \cos \left(m \theta-(s-k) \frac{\pi}{2}\right) d \theta
$$

Если функиия $\varphi(\theta)$ имеет на $[0, \pi]$ производную нечетного порядка $\rho$, то

$$
\begin{gathered}
\int_{0}^{\pi} \varphi(\theta) \sin m \theta d \theta=\sum_{k=0}^{(\rho-1) / 2}(-1)^{k} m^{-2 k-1}\left(\varphi^{(2 k)}(0)-(-1)^{m} \varphi^{(2 k)}(\pi)\right) \\
+(-1)^{(\rho-1) / 2} m^{-\rho} \int_{0}^{\pi} \varphi^{(\rho)}(\theta) \cos m \theta d \theta
\end{gathered}
$$

Для любой функиии $\varphi(\theta)$ на $[0, \pi]$

$$
\int_{0}^{\pi} \varphi(\theta) \cos m \theta d \theta=-\frac{1}{2} \int_{0}^{\pi}(\widetilde{\Delta}(h) \varphi(\theta)) \sin m \theta d \theta
$$

əде $h=\theta u t=\pi /(2 m)$ для $\theta \in(0, \pi /(2 m)) ; h=\pi /(2 m) u t=\theta$ для $\theta \in[\pi /(2 m)$, $\pi-\pi /(2 m)) ; h=\pi-\theta$ u $t=\pi-\pi /(2 m)$ для $\theta \in[\pi-\pi /(2 m), \pi) ;$ во всех случаях $0<h \leqslant \pi /(2 m)$.

ДокАЗАТЕЛЬСтво. В условиях равенства (А) подьнтегральная функция четна; учитывая это, получаем

$$
\begin{aligned}
\int_{0}^{\pi} \varphi(\theta) \cos \left(m \theta-\frac{s \pi}{2}\right) d \theta= & \frac{1}{2} \int_{-\pi}^{\pi} \varphi(\theta) \cos \left(m \theta-\frac{s \pi}{2}\right) d \theta \\
= & \frac{1}{4}\left(\int_{-\pi}^{\pi} \varphi\left(\theta+\frac{\pi}{2 m}\right) \cos \left(m \theta-(s-1) \frac{\pi}{2}\right) d \theta\right. \\
& \left.+\int_{-\pi}^{\pi} \varphi\left(\theta-\frac{\pi}{2 m}\right) \cos \left(m \theta-(s+1) \frac{\pi}{2}\right) d \theta\right) \\
= & \frac{1}{4} \int_{-\pi}^{\pi}\left(\widetilde{\Delta}\left(\frac{\pi}{2 m}\right) \varphi(\theta)\right) \cos \left(m \theta-(s-1) \frac{\pi}{2}\right) d \theta \\
= & \frac{1}{2} \int_{0}^{\pi}\left(\widetilde{\Delta}\left(\frac{\pi}{2 m}\right) \varphi(\theta)\right) \cos \left(m \theta-(s-1) \frac{\pi}{2}\right) d \theta .
\end{aligned}
$$

Последнее равенство обусловлено четностью подынтегральной функции: разновидность функции поменяла ее четность, сдвиг косинуса на $\pi / 2$ поменял его четность.

Применив полученное равенство $k$ раз, придем к равенству (А).

Равенство (В) доказывается последовательным интегрированием по частям. 
Для доказательства равенства (C) применим равенство (А) к четному $2 \pi$-периодическому продолжению $\widetilde{\varphi}$ функции $\varphi$ с $[0, \pi)$ :

$$
\begin{aligned}
\int_{0}^{\pi} \varphi(\theta) \cos m \theta d \theta= & -\frac{1}{2} \int_{0}^{\pi}\left(\widetilde{\Delta}\left(\frac{\pi}{2 m}\right) \widetilde{\varphi}(\theta)\right) \sin m \theta d \theta \\
= & -\frac{1}{2}\left(\int_{0}^{\pi /(2 m)}\left(\varphi\left(\theta+\frac{\pi}{2 m}\right)-\varphi\left(-\theta+\frac{\pi}{2 m}\right)\right) \sin m \theta d \theta\right. \\
& +\int_{\pi /(2 m)}^{\pi-\pi /(2 m)}\left(\widetilde{\Delta}\left(\frac{\pi}{2 m}\right) \varphi(\theta)\right) \sin m \theta d \theta \\
& \left.+\int_{\pi-\pi /(2 m)}^{\pi}\left(\varphi\left(2 \pi-\theta-\frac{\pi}{2 m}\right)-\varphi\left(\theta-\frac{\pi}{2 m}\right)\right) \sin m \theta d \theta\right) \\
= & -\frac{1}{2} \int_{0}^{\pi} \widetilde{\Delta}(h) \varphi(t) \sin m \theta d \theta .
\end{aligned}
$$

Это равенство (C).

ОПредЕЛЕния. Пусть $S^{(r)}(t) f$ - производная по норме $\|\cdot\|$; обозначим

$$
\omega\left(\delta, S^{(r)} f\right)=\sup _{0<h<\delta} \sup _{t}\left\|\Delta(h) S^{(r)}(t) f\right\|,
$$

где $\Delta(h) \varphi(t)=\varphi(t+h)-\varphi(t)$.

ТЕоремА 5. Если существует производная $S^{(r)}(t) f$, то

$$
c\left\|f-D_{n}(f)\right\| \leqslant \omega\left(\frac{\pi}{n+1}, S^{(r)} f\right)+\frac{1}{n+1} \sum_{i=0}^{(r-1) / 2}\left(\left\|S^{(2 i)}(0) f\right\|+\left\|S^{(2 i)}(\pi) f\right\|\right) .
$$

Для $d=3$

$$
\left\|f-D_{n}(f)\right\| \leqslant C \omega\left(\frac{\pi}{n+1}, S^{\prime} f\right)
$$

(в этом случае теоремы 4 и 5 по предложсению автора доказаны Л. В. Егоровым). Константы $C$ ис> 0 не зависят от $n u f$.

ДокАЗАТЕЛЬСТво. Дифференцируем функцию (26) $r$ раз:

$$
\Phi^{(r)}(\theta)=\sum_{s=0}^{r} C_{r}^{s}(S(\theta) f-f)^{(r-s)}\left(\cos \left(\frac{\theta}{2}\right) \sin ^{r-1} \theta\right)^{(s)} .
$$

Поскольку

$$
\sin ^{m} \theta=(2 i)^{-m}\left(e^{i \theta}-e^{-i \theta}\right)^{m}=\sum_{k=0}^{m} a_{k} e^{i((m-2 k) \theta-m \pi / 2)}
$$

(за константами не следим), то

$$
\cos \left(\frac{\theta}{2}\right) \sin ^{m} \theta=\operatorname{Re} e^{i \theta / 2} \sin ^{m} \theta=\sum_{k=0}^{m} a_{k} \cos \left(\left(m-2 k+\frac{1}{2}\right) \theta-\frac{m \pi}{2}\right) .
$$


Тогда

$$
\left(\cos \left(\frac{\theta}{2}\right) \sin ^{r-1} \theta\right)^{(s)}=\sum_{k=0}^{r-1} b_{k} \sin \left(\left(r-2 k-\frac{1}{2}\right) \theta-(r-s) \frac{\pi}{2}\right)
$$

(перешли от $\cos (x-(r-1) \pi / 2)$ к $-\sin (x-r \pi / 2)$ и взяли производную по формуле $\left.(\sin x)^{(s)}=\sin (x+s \pi / 2)\right)$.

Далее,

$$
\begin{aligned}
& \left(\cos \left(\frac{\theta}{2}\right) \sin ^{r-1} \theta\right)^{(s)} \sin \left(\left(n+\frac{1}{2}\right) \theta-\frac{r \pi}{2}\right) \\
& =\sum_{k=0}^{r-1} \frac{b_{k}}{2}\left(\cos \left((n-r+2 k+1) \theta-\frac{s \pi}{2}\right)-\cos \left((n+r-2 k) \theta-r \pi+\frac{s \pi}{2}\right)\right) \\
& =\sum_{j=-r+1}^{r} c_{j} \cos \left((n+j) \theta-\frac{s \pi}{2}\right)
\end{aligned}
$$

(в последнем равенстве перешли от $\cos (x-r \pi+s \pi / 2)$ к $\cos (x-s \pi / 2))$.

Подставляя (29) в (28), затем полученное в (27), учитьвая (30), находим

$D_{n}(f)-f=\varkappa_{n}\left(n+\frac{1}{2}\right)^{-r} \sum_{s=0}^{r} \sum_{j=-r+1}^{r} a_{s j} \int_{0}^{\pi}(S(\theta) f-f)^{(r-s)} \cos \left((n+j) \theta-\frac{s \pi}{2}\right) d \theta$.

При $s=2 i-1>0$ от $\cos (m \theta-s \pi / 2)$ в интегралах (31) переходим к $\sin m \theta$; при $s=2 i>0$ интегралы преобразуем по формуле

$$
\int_{0}^{\pi}(S(\theta) f)^{(r-2 i)} \cos (n+j) \theta d \theta=-\frac{1}{n+j} \int_{0}^{\pi}(S(\theta) f)^{(r-2 i+1)} \sin (n+j) \theta d \theta
$$

опять считаем пока $n+j \neq 0$. Таким образом, с $\cos$ останется интеграл при $s=0$. Его заменим согласно равенству (C) леммы интегралом

$$
\int_{0}^{\pi} \widetilde{\Delta}(h) S^{(r)}(\theta) f \sin (n+j) \theta d \theta
$$

где $0<h \leqslant \pi /(2|n+j|)$. К оставшимся интегралам с $\sin$ и с четными производными $(S(\theta) f)^{(r-2 i+1)}$ снова применим лемму, ее равенство (В) при $\rho=2 i-1$ :

$$
\begin{gathered}
\int_{0}^{\pi}(S(\theta) f)^{(r-2 i+1)} \sin (n+j) \theta d \theta=(-1)^{i-1}(n+j)^{-2 i+1} \int_{0}^{\pi} S^{(r)}(\theta) f \cos (n+j) \theta d \theta \\
+\sum_{k=0}^{i-1}(-1)^{k}(n+j)^{-2 k-1}\left(S^{(r-2 i+1+2 k)}(0) f-(-1)^{m+j} S^{(r-2 i+1+2 k)}(\pi) f\right) .
\end{gathered}
$$

При $r-2 i+1=r-s=0$ в представлении (31) под интегралом стоит функция $S(\theta) f-f$, и поэтому в $(33)$ слагаемое с $k=0$ будет содержать разность $S(\pi)-f$ вместо $f-(-1)^{n+j} S(\pi) f$. 
Последний интеграл в (33) заменим интегралом (32) согласно равенству (В) леммы. При $j=-n$ интегралы в (31) равны нулю при $s$ нечетном; при четном $s$ имеем

$$
\int_{0}^{\pi}(S(\theta) f)^{(r-s)} d \theta=S^{(r-s-1)}(\pi) f-S^{(r-s-1)}(0) f .
$$

Здесь, как и вьше в (33), производные четных порядков.

Переходя в (31) к нормам и используя вышеприведенные представления, получим

$$
\begin{aligned}
\left\|f-D_{n}(f)\right\| \leqslant & \sum_{\substack{j=-r+1 \\
j \neq-n}}^{r} c_{s j} \omega\left(\frac{\pi}{|n+j|}, S^{(r)} f\right) \\
& +|n+j|^{-1} \sum_{\rho=0}^{(r-1) / 2}\left(\left\|S^{(2 \rho)}(0) f\right\|+\left\|S^{(2 \rho)}(\pi) f\right\|\right) .
\end{aligned}
$$

Замена $j$ на 1 осуществляется известным способом (см. ниже).

Если $d=3$, то в (33) будет одно слагаемое с $k=0$, paвное $(1 /(n+j))(S(\pi) f-f)$. Его оценим по норме следующим образом:

$$
\begin{aligned}
\|S(\pi) f-S(0) f\| & =\frac{1}{2}\|S(\pi)-2 S(0) f+S(-\pi)\| \leqslant \pi \omega\left(\pi, S^{\prime} f\right) \\
& \leqslant \pi(n+1) \omega\left(\frac{\pi}{n+1}, S^{\prime} f\right) .
\end{aligned}
$$

Здесь использованы следующие известные в теории приближения функщий свойства модулей непрерывности:

$$
\begin{gathered}
\left\|\widetilde{\Delta}^{2}(h) \varphi\right\| \leqslant 2 h \omega\left(h, \varphi^{\prime}\right), \quad \omega(n \delta, \varphi) \leqslant n \omega(\delta, \varphi), \\
\omega(\eta, f) \leqslant\left(\left[\frac{\eta}{\delta}\right]+1\right) \omega(\delta, f) .
\end{gathered}
$$

\section{СПИСОК ЦИТИРОВАННОЙ ЛИТЕРАТУРЫ}

[1] Градштейн И. С., Рыжик И. М. Таблицы интегралов, сумм, рядов и произведений. М.: Физматгиз, 1963.

[2] Тиман А.Ф. Теория приближения функций действительного переменного. М.: Физматгиз, 1960. 